\title{
Syndecan Regulates Cellular Morphogenesis in Cooperation with the Netrin Guidance Pathway and Rho-family GTPases
}

\author{
Raphaël Dima ${ }^{1, \#}$, Marianne Bah Tahé ${ }^{1, \#}$, Yann A. Chabi ${ }^{1}$, Lise Rivollet ${ }^{1}$, Anthony F. Arena ${ }^{2}$, \\ Alexandra M. Socovich ${ }^{2}$, Daniel Shaye ${ }^{2, *}$, Claire Y. Bénard ${ }^{1,3, *}$
}

Affiliations:

${ }^{1}$ Université du Québec à Montréal

Department of Biological Sciences

141 President Kennedy Avenue

Montréal, QC H2X 1Y4, Canada

${ }^{2}$ University of Illinois-Chicago

Department of Physiology and Biophysics

835 S. Wolcott St, Chicago

IL 60612, USA

${ }^{3}$ University of Massachusetts Medical School

Department of Neurobiology

364 Plantation St, Worcester

MA 01605, USA

\# Equal contribution

${ }^{*}$ Corresponding authors:

Claire Bénard

Université du Québec à Montréal

Department of Biological Sciences

141 President Kennedy Avenue

Montréal, QC H2X 1Y4, Canada

E-mail: benard.claire@uqam.ca ;

claire.benard@umassmed.edu

Phone: 1-514-987-3000, extension -6192

Daniel Shaye

University of Illinois at Chicago - College of Medicine

Department of Physiology and Biophysics (MC901)

835 S. Wolcott Ave, MSB Room E202

Chicago, IL 60612, USA

E-mail: shaye@uic.edu

Phone: +1-312-413-0492 


\section{ABSTRACT}

The establishment of complex cell shapes is essential for specific cellular functions, and thus critical in animal development and physiology. Heparan sulfate proteoglycans (HSPGs) are conserved glycoproteins that regulate interactions between extracellular signals and their receptors, to orchestrate morphogenetic events and elicit cellular responses. Although HSPG-regulated pathways have been implicated in regulating the guidance of neuronal migrations, whether HSPGs regulate earlier aspects of cellular development that dictate cell shape remains unknown. HSPGs consist of a protein core (e.g., Syndecan, Perlecan, Glypican, etc.) with attached heparan sulfate (HS) glycosaminoglycan chains, which are synthesized by glycosyltransferases of the exostosin family. Using mutations in the two C. elegans HS glycosyltransferases genes, rib-1 and rib-2, we reveal that HSPGs control the number of cellular projections in the epithelial excretory canal cell, which can form more than its normal four canals in these mutants. We identify SDN-1/Syndecan as the key HSPG that regulates the number of excretory canal cell projections in a cell-autonomous manner. We also find that Syndecan and guidance receptors for Netrin function in the same pathway to restrict the number of cellular projections. Furthermore, we show that the formation of extra projections in the absence of Syndecan requires the conserved Rho-family GTPases CED-10/Rac and MIG-2/RhoG. Our findings not only contribute to understanding the roles of conserved HSPGs in cellular morphogenetic events, but also reveal the existence of an HSPG-regulated system operating to guarantee that a precise number of cellular projections is established during cell development. Given the evolutionary conservation of developmental mechanisms and the molecules implicated, this work provides information relevant to understanding the cellular and molecular bases of the development of precise cellular morphologies in varied cell types across animals.

Keywords: cell morphology, HSPG, Syndecan, Rho-GTPases, CED-10/Rac, MIG-2/RhoG 


\section{INTRODUCTION}

The establishment of complex cell shapes is essential for specific cellular functions, and thus critical is in animal development and physiology. Certain cells possess a specialized morphology that allow distinct functions such as the flow of information along a neuron, or the osmoregulation carried out by the tubular cells of the excretory system. Whereas the mechanisms driving the outgrowth and guidance of neuronal projections have been uncovered and described over the past two decades, how the number of cellular projections is controlled during development remains poorly understood.

Cellular morphogenesis relies on mechanisms intrinsic and extrinsic to cells $\mathbf{s}^{1,2}$, which bring about asymmetries within the developing cell and establish dynamic motile structures underlying oriented growth. In developing neurons, for instance, growth cones bear multiple receptors for extracellular guidance cues such as UNC-6/Netrin ${ }^{3}$. UNC-6/Netrin is a molecule with a key role in modulating the outgrowth and guidance of the extending growth cone, as well as of other polarized cells ${ }^{4}$. The UNC-6/Netrin signal can act as a growth cone attractant by interaction with a homodimer of its receptor UNC-40/DCC ${ }^{4-6}$, or as a repellent by interaction with an heterodimer of UNC-40/DCC and UNC-5/UNC5 receptors ${ }^{7}$ inducing destabilization of the cytoskeleton in the growth cone protrusions ${ }^{8,9}$. The UNC-6/Netrin guidance pathway is also involved cell symmetry breakage and the definition of the axon formation site, prior to its roles in the guidance of established cellular projections ${ }^{2}$.

Receptors of guidance cues recruit and activate intracellular signals to induce cell responses, which often involve cytoskeleton rearrangements. MIG-2/RHOG and CED-10/Rac are two small Rho-GTPases members of the Rac-like GTPases subfamily that function in the UNC-6/Netrin guidance pathway, to regulate the growth cone's cytoskeleton dynamics ${ }^{10}$. MIG-2/RHOG and CED-10/Rac are also required for proper extension outgrowth of other cells such as those of the excretory canal cell in C. elegans ${ }^{11}$. Besides their role in guidance, these regulators also modulate the cytoskeleton during the earliest phase of cell extension or axonal specification ${ }^{12}$.

Heparan sulfate proteoglycans (HSPGs) are conserved glycoproteins that regulate interactions between extracellular signals and their receptors, to elicit cellular responses and orchestrate morphogenesis. They function as co-receptors, modulating the interaction of morphogens and guidance cues with their receptor, in numerous developmental pathways such as that of netrin ${ }^{13,14}$, among others ${ }^{15}$. HSPGs are composed of a core protein to which heparan sulphate (HS) glycosaminoglycan chains are attached. HS chains are synthesized by exostosin glycosyltransferases. The HSPGs core proteins ${ }^{13,15}$ as well as their HS chains can both play roles in the development of neurons and other cells with complex morphologies ${ }^{15-17}$, and the chemical modification of HS chains increases their specificity of function ${ }^{15,18}$.

Here, we uncover a mechanism that regulates the number of cellular extensions established by a cell during development. Using the excretory cell of $C$. elegans, we reveal that HSPGs, and more specifically, SDN-1/Syndecan is the key HSPG at the center of this mechanism. We find SDN-1 functions in a cellautonomous manner together with the Netrin guidance receptors to restrict the number of cellular projections. Furthermore, we show that the formation of extra cellular projections in the absence of Syndecan requires the conserved Rho-family GTPases CED-10/Rac and MIG-2/RhoG. Together, our findings contribute to understanding the roles of conserved HSPGs in cellular morphogenetic events, in mechanism regulating the development of precise cellular morphologies.

\section{MATERIALS AND METHODS \\ Nematode strains and genetics}

Nematode cultures were maintained in an incubator at $20^{\circ} \mathrm{C}$ (unless otherwise noted) on NGM plates seeded with Escherichia coli OP50 bacteria as described ${ }^{19}$. Alleles used in this study are listed in Table S3. Strains were constructed using standard genetic procedures and are listed in Table S2. Genotypes were confirmed by genotyping PCR or by sequencing when needed. Primers used to build strains are listed in Table S1. All 
the mutant alleles and reporter strains were outcrossed with the Bristol N2 wild-type strain at least 3 times prior to use for analysis or strain building.

\section{Microinjections and transgenic animals}

Transgenic animals were generated by standard microinjection techniques ${ }^{20}$. Each plasmid was injected at 0.2 $\mathrm{ng} / \mu \mathrm{L}$ (pCB420), $1 \mathrm{ng} / \mu \mathrm{L}$ (pCB427), $5 \mathrm{ng} / \mu \mathrm{L}$ (pCB436), 10ng/ $\mathrm{LL}$ (pCB423) or $25 \mathrm{ng} / \mu \mathrm{L}$ (pCB242, pCB265, pCB425) as indicated in Table S5, with the co-injection marker Punc-122::rfp (coelomocytes in red) at a concentration of $50 \mathrm{ng} / \mu \mathrm{L}$. $\mathrm{pBSK}+$ was used to increase the total DNA concentration to $200 \mathrm{ng} / \mathrm{\mu L}$.

\section{Microscopy and excretory canal cell observations}

Worms were grown in an incubator at $20{ }^{\circ} \mathrm{C}$ for at least 3 generations prior to analysis. Worms were anesthetized with $75 \mathrm{mM} \mathrm{NaN}_{3}$ between a coverslip and a $5 \%$ agarose pad. Observations were made using a x40 or x63 objective on a Zeiss Axio Imager (M2) fluorescence microscope. Images of representative phenotypes were captured using the Zeiss AxioCam camera and the Zen software. Images of embryos were deconvoluted using the software AutoQuant X3 for better resolution.

The excretory canal cell and its canals were examined in L4 stage larvae, unless otherwise specified, using the transgenic reporters arls164 (Pglt-3::venus) or bgls312 (Ppes-6::gfp). The excretory canal cell is located near and ventral to the terminal bulb of the pharynx in the head and bears four lateral canals in the wildtype: two anterior canals located along each side of the animal's head (one on the left and one on the right side), and two posterior canals located along the length of the animal's body (one on the left side of the animal and one on the right side). An animal was considered to have supernumerary canals only when more than four canals had grown directly out of the excretory canal cell soma, regardless of the canal's position in the animal's body (left, right, dorsal, ventral, anterior or posterior). Only canals that (1) stem from the excretory cell soma and (2) are longer than the diameter of the excretory cell soma ( $25 \mu \mathrm{m}$ in length) were counted as canals. Thus, branches that had extend along the length of a canal were not counted as an extra canal, as they do not arise from the excretory canal cell soma. Other types of defects were observed during our analyses such as missing canals, misguided canals, and shorter canals, as described elsewhere ${ }^{4,21-23}$, which were not counted as defective in this study as we focused on the presence of supernumerary canals.

\section{Rescue and expression constructs}

Numbers in square brackets below refer to position along the cDNA.

Pmec-7::sdn-1 cDNA (pCB242): The cDNA of sdn-1 was amplified from yk139f3 and ligated into a Pmec-7 containing pPD96.41 vector using enzymes Xmal - Xhol as described previously ${ }^{13}$.

Pglt-3::sdn-1 cDNA (pCB420): Vector pCB242 (Pmec-7::sdn-1 cDNA) was digested with Sphl [11] and Xmal [888] to release Pmec-7 and ligated with insert Pglt-3 [303 bp] amplified from vector pDS629 (Pglt-3::zif1) using primers oCBQc48 (GCTTGCATGCTTTCGAATCG) and oCBQc49 (CATGATCCCGGGTATGGATCCGGTACCTCC) to add on Xmal site, and then digested with Sphl [9] and Xmal [292].

Pgrd-10::sdn-1 cDNA (pCB265): The cDNA of sdn-1 was amplified with primers oCB1032 and oCB1033, digested with EcoRI and Agel and ligated into grd-10::GFP containing pPD95.75 backbone vector with GFP replaced by the cDNA of $s d n-1$ with EcoRI and Agel.

Prgef-1::sdn-1 cDNA (pCB425): Vector pCB199 (Prgef-1::rib-1 cDNA) was digested with Xmal [3480] and Apal [5441] to release the CDNA of rib-1 and the unc-54 3'UTR and ligated with the fragment containing the sdn-1 cDNA and unc-54 3'UTR amplified from pCB242 (Pmec-7::sdn-1 cDNA) digested with Xmal [888] and Apal [2734].

Pmyo-3::sdn-1 cDNA (pCB423): Vector pCB332 (Pmyo-3::/on-2 cDNA) was digested with Xbal [2414] and Sacl [3998] to release the cDNA of lon-2 and ligated with the fragment containing the cDNA of $s d n-1$ 
amplified from $\quad$ (Pglt-3::sdn-1 cB420 cDNA) using primers oCB1715 (CATGATTCTAGACCATACCCGGGATGATTCTG) and oCB1716 (AAGATCTCGGGAGCTCCTC) to add on $X$ bal site and then digested with Xbal [7] and Sacl [901].

Prab-3::sdn-1 cDNA (pCB436): Vector pCB428 (Prab-3::sax-7 cDNA) digested with Xmal [1528] and $X$ hol [5091] to release the CDNA of sax-7 and ligated with the fragment containing the cDNA of sdn-1 amplified from pCB420 (Pglt-3::sdn-1 cDNA) [4737 bp] digested with Xmal [294] and Xhol [1167].

Pmyo-2::sdn-1 cDNA (pCB427): Vector pCB242 (Pmec-7::sdn-1 cDNA) was digested with Xbal [25] and Xmal [888] to release Pmec-7 promoter and ligate with insert Pmyo-2 [863 bp] amplified from pCB230 (Pmyo-2::rib-2 cDNA) digested with Xbal [25] and Agel [1309].

All inserts of finalized clones were verified either by sequencing for cloned PCR amplicons, or by restriction digests and PCR amplification for fragments released from an already sequenced vector.

\section{RESULTS}

\section{The HSPG SDN-1/Syndecan limits the number of excretory canal cell projections}

The excretory canal cell, which ensures osmoregulation in C. elegans, is an epithelial tubular cell whose soma is located in the head of the animal, and from which four projections or canals extend along the body sides, two anteriorly and two posteriorly (Fig. 1A, top panel). During previous analyses, we noticed excretory canal cell abnormalities in mutants affecting the heparan sulphate $(\mathrm{HS})$ chains biosynthesis ${ }^{16}$. HS chain biosynthesis is catalyzed by a co-polymerase composed of two exostosin glycolsyltransfereases RIB-1/EXT1 and RIB2/EXTL3 in C. elegans and mammals alike (Fig. 1B). In hypomorphic viable mutants rib-1(qm32) and rib2(qm46), the guidance of excretory canal cell projections, neurons, axons, and the distal tip cell, is defective ${ }^{16}$. The outgrowth per se of the excretory canal cell projections can also be affected in these mutants, as the excretory canals can be short (Fig. 1A). These mutants also frequently exhibit a striking and novel type of defect, where animals can have an excessive number of excretory canals, compared to the wild type. We characterized this defect using a reporter to visualize the excretory cell (Ppes-6::gfp) and found that $\sim 27 \%$ of the rib-1 and rib-2 mutants develop supernumerary canals, ranging from five to eight excretory canals, compared to the four projections invariably observed in the wild type (Fig. 1C). Supernumerary excretory canals as long as the normal excretory canals, or shorter, located anteriorly or posteriorly from the soma of the excretory canal cell, or even in a ventral or lateral position were observed. Excretory canal cell projections or canals that grew from the soma of the excretory canal cell and that exceeded the set of four normally present in the wild type, are henceforth called "extra-canals" or "supernumerary canals". In contrast, branches extending from an excretory canal were not considered to be an excretory canal, as they are secondary extensions not growing directly from the soma. The outgrowth and guidance defects of the excretory canals of HSPGs or other mutants have been studied elsewhere ${ }^{4,21-23}$. Here we focus on studying the uncommon supernumerary excretory canals defect, as it offers the unique opportunity to investigate the mechanisms that restrict the number of cellular extensions. The excretory cell is an ideal model to address this question as its development has been characterised in detail ${ }^{11,24,25}$, and its outgrowth and guidance are regulated by pathways shared with neurons s,23,26. $^{4}$.

Having observed defects in the number of cellular projections in HS chain biosynthesis mutants, we next examined whether HS chain modification may be important in this process. Indeed, subsequent to their polymerization, HS chains are chemically modified, which impacts their role in neuronal migration and guidance ${ }^{15,27-29}$. We tested whether HS chain modifications carried out by the $C$. elegans sulfotransferases HST-2/HS2ST1 and HST-6/HS6ST1 and the epimerase HSE-5/GLCE may affect the number of excretory canals, by examining single null mutants for each of these enzymes, as well as triple mutant animals using the reporter Pglt-3::venus. We found that animals mutant for $h s e-5$, $h s t-2$ or $h s t-6$ develop extra canals (Fig. 1D), indicating that the HS chains modifications may play a role here. We constructed a triple mutant strain and detected no enhancement of the defect in animals mutant for all three genes $h s e-5, h s t-2$ or $h s t-6$, suggesting 
that all these HS modifications affect the development of the excretory cell in a similar way (Fig. 1D). Taken together these results suggest that HS chain biosynthesis and modification are required for the establishment of the proper number of canals in the excretory canal cell.

Disruption of the HS co-polymerase genes rib-1 and rib-2, or of the HS modifying enzymes, affects the biosynthesis of all HSPGs simultaneously. To determine which HSPG(s) specifically act(s) to regulate the number of excretory canals, we systematically examined null or strong loss-of-function mutations that disrupt each of the HSPGs individually. Six HSPGs are known in C. elegans, namely UNC-52/Perlecan, AGR-1/Agrin, CLE-1/Collagen type XVIII, LON-2/Glypican, GPN-1/Glypican, and SDN-1/Syndecan. We found that among the HSPG core-protein mutants tested (Fig. 1E), only sdn-1(zh20) null mutants showed a significant number of extra canals ( $46 \%$ of the mutants; Fig. 1E) compared to wild type. Like for rib-1 and rib-2 mutants, the number and position of the extra canals vary in sdn-1 mutants. A similarly penetrant defect was observed in animals mutant for a second allele of $s d n-1$, the strong loss-of-function $s d n-1$ (ok449) (46\%, Fig. 1E). Thus, the HSPG SDN-1/Syndecan limits the number of excretory canal cell projections.

\section{Wild-type and supernumerary canals display similar temporal and sub-cellular characteristics}

At $\sim 400$ min of embryonic development, the soma of the excretory canal cell undergoes lumen formation and two lateral extensions start projecting ${ }^{24}$ (Fig. 2A). At the 2-fold stage, these lateral extensions bifurcate to give rise to the four lateral canals, generating the cell's typical $\mathrm{H}$ shape. These canals lengthen and their lumen extends along the length of the canals, until they span the entire length of the animal ${ }^{24,26,30}$. To determine when the supernumerary canals form in sdn-1 mutants, we examined wild-type and sdn-1(zh20) mutant embryos at the time of canal formation ( 3 -fold stage $\approx 550 \mathrm{~min}$ of development). No extra canals are ever seen in wild-type embryos at this time or later; on the other hand, supernumerary canals are already present in sdn-1 mutant embryos (Fig. 2B). Like normal canals, the supernumerary canals extend from the soma of the excretory canal cell, at the same time as normal canals, indicating that the mechanism at play acts during the initial development of the cell to regulate the number of cellular projections stemming from the soma. Furthermore, the penetrance of the defects in sdn-1(zh20) mutant animals is stable between the first (L1) and fourth (L4) larval stages (Fig. 2C), consistent with the notion that $s d n-1 /$ Syndecan functions during the initial development of the excretory canal cell to control canal number.

To address whether the additional cellular projections are bona fide canals, we examined actin organization in the excretory cell using a life-Act transgene fused to the marker tagRFP (expressed specifically in the excretory canal cell). In wild-type animals, actin is present at the terminal web lining under the apical membrane along the lumen of the canals, surrounded by a cytoplasmic marker expressed in the excretory canal cell ${ }^{23}$ (Pglt-3::CFP; Fig. 2D). Actin was similarly organized in the supernumerary canals of the sdn1(zh20) mutant animals (Fig. 2D). Thus, the supernumerary canals observed in sdn-1/Syndecan mutants display similar temporal and sub-cellular characteristics to wild-type canals.

\section{Syndecan functions cell-autonomously to restrict the number of cell projections in the excretory canal cell}

In a $\sim 500$ min old wild-type embryo (Wormatlas ${ }^{31,32}$, Fig. 3A), when the excretory canals start projecting, the excretory cell is in direct contact with the pharynx and neurons, and in close proximity with the body wall muscles. Later on, the canals line up close to lateral epidermal seam cells. To address where SDN-1/Syndecan functions to regulate canal number, we tested where its expression may suffice for normal excretory canal cell development. For this, we generated transgenic sdn-1(zh20) animals that express wild-type copies of $s d n-1(+)$ under several cell-specific promoters and analysed their capacity to rescue the extra canal defects of sdn1(zh20) mutants. Expression in cells near the excretory cell such as the seam cells, body wall muscles, pharyngeal muscles, 6 mechanosensory neurons, or pan-neuronal expression did not rescue the defects of the sdn-1(zh20) mutants (Fig. 3B). Also, the two CAN neurons, which are in close proximity with the excretory 
canals all along the body sides and are physiologically related ${ }^{33}$, do not impact canal development since unc39(e257) mutants, which are strongly defective in CAN cell migration and axon pathfinding ${ }^{34}$, have normal excretory canals $(0 \%, \mathrm{~N}=119)$. In contrast, expression of wild-type copies of $s d n-1(+)$ specifically in the excretory cell (transgene Pglt-3::sdn-1(+)) strongly rescued the defects of the sdn-1(zh20) mutants (Fig. 3B), indicating that $s d n-1$ functions in the excretory cell. The gene $s d n-1$ is expressed in the excretory cell during its development ${ }^{35}$ (Fig. S1A), consistent with SDN-1/Syndecan functioning cell-autonomously to restrict the number of cell projections in the excretory canal cell.

\section{Syndecan and Netrin function in same pathway to restrict the number of cell projections in the excretory canal cell}

To gain insight into the mechanism controlling cell projection number, we determined whether pathways known to guide the migration of developing canals ${ }^{4}$ might also function to regulate canal number. Hedgcock and colleagues anecdotally reported supernumerary canals ${ }^{4}$ in mutants for unc-6/Netrin and its receptors. UNC6/Netrin signals can be attractive in cell migration and/or axon pathfinding (Fig. 4A), in which case UNC-6 interacts with two UNC-40 receptors, inducing cytoskeleton rearrangements to orient migration towards the UNC-6 signal ${ }^{4-6}$. UNC-6/netrin signals can also be repulsive, where UNC-6 links a heterodimer of an UNC-40 receptor and an UNC-5 receptor, leading to migration away from the UNC-6/netrin signal ${ }^{7}$ (Fig. $4 A$ ). We thus tested whether the guidance cue UNC-6/Netrin and its receptors UNC-40/DCC and UNC-5/UNC5, are involved in the regulation of excretory canal number. Loss of function of unc-6, with either the null mutation unc-6(ev400) or the hypomorphic mutation unc-6(e78), leads to supernumerary canals on the excretory cell (Fig. 4B and C). Furthermore, loss of function of either of the unc-6/netrin receptors, using single null mutations unc40(e1430), unc-40(e271) or unc-5(e53), also results in supernumerary canals (Fig. 4B and C), and the simultaneous loss of both receptors in unc-40(e1430) unc-5(e53) double mutants does not enhance the defects, pointing to their action in the same mechanism to control cell projection number in the excretory canal cell. Taken together, these results indicate that the netrin guidance cue as well as its main receptors UNC40/DCC and UNC-5/UNC5, are involved in a mechanism controlling the number of cellular extensions developed.

To ask whether the unc-6/netrin receptors contribute to the same mechanism as $s d n-1 /$ Syndecan in controlling cell projection number of the excretory canal cell, we analysed their genetic interactions using null alleles. Simultaneous loss of $s d n-1$ and either unc-5 or unc-40, in the double mutants sdn-1(zh20); unc-5(e53) or sdn-1(zh20); unc-40(e1430) did not enhance the supernumerary canals phenotype (Fig. 4D). This is consistent with the notion that $s d n-1 / S y n d e c a n$ and the two guidance receptors function in the same pathway and suggests that they may participate in a common mechanism with SDN-1/Syndecan to regulate the number of cell projections.

\section{MIG-2/RhoG and CED-10/Rac are required for the formation of extra projections upon SDN-1/Syndecan loss}

The Rho-GTPases MIG-2/RhoG and CED-10/Rac are molecular switches that control cytoskeleton dynamics during embryonic morphogenesis ${ }^{11}$ and axonal pathfinding ${ }^{10}$, playing roles in the UNC-6/Netrin pathway and cooperating with HSPGs like Syndecan in mammal cells ${ }^{36-38}$. To investigate the role of these Rho-GTPases in the control of cell projection number, we used mutations in these genes. Single mutant animals with the partial loss-of function mutation ced-10(n1993) or the null mutation mig-2(mu28) show no supernumerary canals (Fig. 5A,B). The double mutants sdn-1(zh20); ced-10(n1993) and mig-2(mu28) sdn-1(zh20), however, both display a reduced number of supernumerary canals compared to the single mutant animals sdn-1(zh20). This suggests that the normal functions of CED-10/Rac and of MIG-2/RhoG promote canal projection, and are required for the formation of extra projections upon SDN-1/Syndecan loss. 


\section{DISCUSSION}

Cells with complex morphologies such as neurons require establishment of precise cell shapes, with a defined number of cellular projections, to enable function. Understanding of outgrowth and guidance of cellular projections has greatly progressed, and HSPGs have emerged as important players in these morphogenetic events $^{13,15,16,39}$. In contrast, the regulation of the number of cellular projections at the very onset of their development is poorly understood. Thanks to a new phenotype where a developing cell has more projections than it should, we were able to identify a mechanism by which the number of extensions established during development is regulated. We show that early in the development of the excretory canal cell HSPG SDN$1 /$ Syndecan acts in a cell-autonomous manner, together with the netrin guidance pathway and two RhoGTPases CED-10/Rac and MIG-2/RhoG to control the number of excretory canals (Fig. 5C).

\section{Study of a novel phenotype: A polarized cell developing supernumerary extensions}

Several defects of the excretory canal cell observed in HSPG mutants were previously studied (shorter or missing canals likely due to outgrowth issues ${ }^{4,11,21,22}$, guidance defects ${ }^{4,16}$ or branched canals ${ }^{22}$ ). Here, we report a new phenotype, which had been anecdotally noted by others previously ${ }^{4,40}$, but remained unstudied. The depletion of HS biosynthesis in rib-1 and rib-2 mutants, the loss of sdn-1/Syndecan, and the disruption of the unc-6/Netrin pathway, all induce supernumerary cellular projections. These supernumerary projections stem directly out of the soma of the excretory canal cell (Fig. 1A) and display temporal and cellular hallmarks of wild-type canals (Fig. 2B). Given that no extra canals were ever seen at that time in wild-type embryos, these supernumerary extensions do not appear to arise from uneliminated transitional protrusions that would later be eliminated in wild-type development, and instead appear to arise as de novo projections when the mechanism to restrict them is abrogated. This is in contrast with the extension of short branches from a preexisting cellular projection ${ }^{16,41}$, such as in aging neurons ${ }^{42-44}$, or the bifurcation of a growth cone leading to multiple extensions ${ }^{45}$. The growth of multiple cellular projections having the hallmarks of mature extensions and growing from a cellular soma has been observed only rarely in cells in culture under specific conditions ${ }^{12}$ and it even more rarely in vivo ${ }^{46}$. Thus, the model described here sets the ground for detailed studies of the molecular and cellular mechanisms at play.

\section{Syndecan restricts the number of canals in the excretory cell}

Our systematic analysis of all available HSPG core protein mutants in C. elegans led to the identification of one key evolutionarily conserved HSPG, SDN-1/Syndecan, as a cell-autonomously acting inhibitor of supernumerary projections (Fig. 1E, Fig. 3B). Whereas shedding of extracellular regions of Syndecan has been reported in other contexts ${ }^{47}$ allowing it to act at a distance, in the mechanism of cellular projection control in the excretory canal cell, Syndecan was found to function in this very cell during development, as only expression in the developing excretory cell restored function, while expression in neighbouring cells did not (Fig. 3B).

HSPGs function can be mediated by either their core protein or their glycosaminoglycan HS chains ${ }^{15}$, particularly during morphogenesis of polarized cells ${ }^{16}$. Mutation of the HS chain polymerisation in rib-1 and rib2 mutants alters the number of cellular projections (Fig. 1C), indicating that either the HS chains per se play a role in this mechanism, or that the level or targeting of the entire HSPG may be affected. Similar penetrance of the supernumerary canal (Fig. 1E) was seen in the null mutant $s d n-1$ (zh20) and the partial mutant sdn1(ok499), which expresses a truncated SDN-1/Syndecan protein lacking the HS chains attachment region ${ }^{48}$. This supports the notion that the HS chains are important for the role of Syndecan in the control of cell projection number, either because they play a direct role in the mechanism as for axonal guidance ${ }^{16,49,50}$, or because the absence of HS chains impacts the level or targeting of SDN-1. Similarly, disruption of HS chain maturation in the triple HS modifying enzymes null mutant hse-5(tm472); hst-2(ok595) hst-6(ok273) showed 
supernumerary cellular projections (Fig. 1D), suggesting that HS chain maturation is important for HSPGs in the regulation of cellular projection number.

\section{Syndecan cooperates with guidance pathways to control the number of cellular projections}

We showed that the guidance cue UNC-6/Netrin and its receptors UNC-40/DCC and UNC-5/UNC5 are part of the mechanism restricting the number of cellular projections of the excretory cell (Fig. 4C). HSPGs have been described as co-receptors mediating interactions of guidance cues with their receptors ${ }^{13,15}$, and here we uncover that SDN-1/Syndecan and UNC-6/Netrin receptors UNC-40/DCC and UNC-5/UNC5 function in same pathway to control cellular projection number in the developing excretory canal cell. Consistent with our findings, a link between Syndecan and the UNC-6/Netrin pathway was suggested in the context of D-type motor axon guidance ${ }^{14}$, distal tip cell migration ${ }^{51}$, and in synaptic development, where the content of UNC$40 /$ DCC at chemical synapses requires SDN-1/Syndecan ${ }^{52}$, reinforcing the notion that Syndecan and the Netrin guidance pathway together underlie key developmental events.

\section{Syndecan controls the number of cell extension through two Rho-GTPases}

Similarities in the cytoskeletal organisation exist between the growth cone of a developing neuron ${ }^{11}$ and the tip of a growing canal, where actin and microtubules need to be tightly regulated for proper development ${ }^{11,25}$. CED-10/Rac and MIG-2/RhoG are two Rho-GTPases that regulate cytoskeletal organisation in numerous biological processes ${ }^{10,11}$. Of particular interest, they regulate the actin cytoskeleton downstream of the UNC$6 /$ Netrin guidance pathways in the growth cone of axons ${ }^{6,53}$. We have showed here that MIG-2 and CED-10 are required for the development of supernumerary cellular projections when the SDN-1-mediated mechanisms to control the number of excretory canals is disrupted, establishing a novel link between these Rho-GTPases and a HSPG in C. elegans. Several scenarios for the role of SDN-1/Syndecan can be envisaged. (1) SDN-1/Syndecan may act as a co-receptor with the UNC-6/Netrin receptor(s), modulating the function of downstream targets of this pathway such as the cytoskeleton regulators CED-10/Rac and MIG2/RhoG, thus controlling the number of extensions formed by the cytoskeleton regulation. (2) SDN-1/Syndecan may directly act on the Rho-GTPases CED-10/Rac and MIG-2/RhoG, as mammalian Syndecans do ${ }^{36-38}$, to modulate the function of the UNC-6/Netrin pathway by regulating UNC-40/DCC plasma membrane localization, as observed in axon development ${ }^{54}$. (3) SDN-1/Syndecan might act as a central regulator, impacting the UNC6/Netrin pathway as well as the cytoskeleton modulators CED-10/Rac and MIG-2/RhoG in distinct ways that converge in the regulation of cell projection number during the development of the excretory canal cell.

Taken together our results uncover a novel developmental mechanism ensuring the control of cellular projection number during the development of highly polarized cells. HSPG SDN-1/Syndecan is central to this mechanism, which also employs the guidance cue UNC-6/Netrin and its main receptors UNC-40/DCC and UNC-5/UNC5 in the same genetic pathway, as well as two Rho-GTPases CED-10/Rac and MIG-2/RhoG. Further studies are needed to address the impact of SDN-1/Syndecan on the regulation of receptor localization and of cytoskeleton dynamics during cell development. Given the evolutionary conservation of developmental mechanisms and the molecules implicated, this work provides information relevant to understanding the cellular and molecular bases of the development of precise cellular morphologies in varied cell types across animals.

\section{ACKNOWLEGMENTS}

We thank Marianne Moore for her technical assistance at the early stages of the project; Denis Flipo (UQAM) for his confocal microscopy expertise; several investigators for their gift of plasmids, including Dr. A. Fire ( $p P D$ clones), Dr. Y. Kohara (yk cDNA clones); Wormbase; the Caenorhabditis Genetics Center, which is funded by $\mathrm{NIH}$ Office of Research Infrastructure Programs (P40 OD010440) for strains; WormAtlas and Wormlmage, which are funded by NIH OD010943 to David H. Hall; John White and Jonathan Hodgkin for donation of the 
MRC/LMB archives to the D. Hall laboratory for curation; as well as Richard Durbin, who did the original TEM work on the embryo, which the Hall laboratory published for him on WormAtlas. This work was supported by the National Science and Engineering Research Council of Canada, the Fond de Recherche du Québec-Santé (C.B. Research Scholar Jr2 and Sr), the Canadian Funds for Innovation-John Evan Leaders Equipment Grant, two Excellence in Doctoral Research Scholarships from the Research Center CERMO-FC (Centre d'excellence de recherche sur les maladies orphelines-Fondation Courtois) to R.D., three Excellence Masters Research Scholarships to M.B.T by the UQAM Fondation.

\section{REFERENCES}

1. Neukirchen, D. \& Bradke, F. Neuronal polarization and the cytoskeleton. Seminars in Cell \& Developmental Biology 22, 825-833 (2011).

2. Adler, C. E., Fetter, R. D. \& Bargmann, C. I. UNC-6/Netrin induces neuronal asymmetry and defines the site of axon formation. Nat Neurosci 9, 511-518 (2006).

3. Ishii, N., Wadsworth, W. G., Stern, B. D., Culotti, J. G. \& Hedgecock, E. M. UNC-6, a laminin-related protein, guides cell and pioneer axon migrations in C. elegans. Neuron 9, 873-881 (1992).

4. Hedgecock, E. M., Culotti, J. G. \& Hall, D. H. The unc-5, unc-6, and unc-40 genes guide circumferential migrations of pioneer axons and mesodermal cells on the epidermis in C. elegans. 4, 61-85 (1990).

5. Chan, S. S.-Y. et al. UNC-40, a C. elegans Homolog of DCC (Deleted in Colorectal Cancer), Is Required in Motile Cells Responding to UNC-6 Netrin Cues. Cell 87, 187-195 (1996).

6. Gitai, Z., Yu, T. W., Lundquist, E. A., Tessier-Lavigne, M. \& Bargmann, C. I. The Netrin Receptor UNC40/DCC Stimulates Axon Attraction and Outgrowth through Enabled and, in Parallel, Rac and UNC115/AbLIM. Neuron 37, 53-65 (2003).

7. Leung-Hagesteijn, C. et al. UNC-5, a transmembrane protein with immunoglobulin and thrombospondin type 1 domains, guides cell and pioneer axon migrations in C. elegans. Cell 71, 289-299 (1992).

8. Norris, A. D., Sundararajan, L., Morgan, D. E., Roberts, Z. J. \& Lundquist, E. A. The UNC-6/Netrin receptors UNC-40/DCC and UNC-5 inhibit growth cone filopodial protrusion via UNC-73/Trio, Rac-like GTPases and UNC-33/CRMP. Development 141, 4395-4405 (2014).

9. Norris, A. D. \& Lundquist, E. A. UNC-6/netrin and its receptors UNC-5 and UNC-40/DCC modulate growth cone protrusion in vivo in C. elegans. Development 138, 4433-4442 (2011).

10. Reiner, D. J. \& Lundquist, E. A. Small GTPases. WormBook: The Online Review of C. elegans Biology [Internet] (WormBook, 2018).

11. Shaye, D. D. \& Soto, M. C. Chapter Six - Epithelial morphogenesis, tubulogenesis and forces in organogenesis. in Current Topics in Developmental Biology (eds. Jarriault, S. \& Podbilewicz, B.) vol. 144 161-214 (Academic Press, 2021).

12. Dupraz, S. et al. RhoA Controls Axon Extension Independent of Specification in the Developing Brain. Current Biology 29, 3874-3886.e9 (2019).

13. Blanchette, C. R., Perrat, P. N., Thackeray, A. \& Bénard, C. Y. Glypican Is a Modulator of NetrinMediated Axon Guidance. PLoS Biol 13, (2015).

14. Gysi, S., Rhiner, C., Flibotte, S., Moerman, D. G. \& Hengartner, M. O. A Network of HSPG Core Proteins and HS Modifying Enzymes Regulates Netrin-Dependent Guidance of D-Type Motor Neurons in Caenorhabditis elegans. PLoS One 8, (2013).

15. Saied-Santiago, K. \& Bülow, H. E. Diverse roles for glycosaminoglycans in neural patterning. Dev. Dyn. 247, 54-74 (2018).

16. Blanchette, C. R., Thackeray, A., Perrat, P. N., Hekimi, S. \& Bénard, C. Y. Functional Requirements for Heparan Sulfate Biosynthesis in Morphogenesis and Nervous System Development in C. elegans. PLOS Genetics 13, e1006525 (2017).

17. Rhiner, C., Gysi, S., Fröhli, E., Hengartner, M. O. \& Hajnal, A. Syndecan regulates cell migration and axon guidance in C. elegans. Development 132, 4621-4633 (2005).

18. Bülow, H. E. \& Hobert, O. The Molecular Diversity of Glycosaminoglycans Shapes Animal Development. Annual Review of Cell and Developmental Biology 22, 375-407 (2006).

19. Brenner, S. The genetics of Caenorhabditis elegans. Genetics 77, 71-94 (1974).

20. Mello, C. \& Fire, A. DNA transformation. Methods Cell Biol. 48, 451-482 (1995). 
21. Lant, B. et al. CCM-3/STRIPAK promotes seamless tube extension through endocytic recycling. Nature Communications 6, 1-13 (2015).

22. Abrams, J. \& Nance, J. A polarity pathway for exocyst-dependent intracellular tube extension. Elife 10, e65169 (2021).

23. Shaye, D. D. \& Greenwald, I. The Disease-Associated Formin INF2/EXC-6 Organizes Lumen and Cell Outgrowth during Tubulogenesis by Regulating F-Actin and Microtubule Cytoskeletons. Developmental Cell 32, 743-755 (2015).

24. Sundaram, M. V. \& Buechner, M. The Caenorhabditis elegans Excretory System: A Model for Tubulogenesis, Cell Fate Specification, and Plasticity. Genetics 203, 35-63 (2016).

25. Buechner, M., Yang, Z. \& Al-Hashimi, H. A Series of Tubes: The C. elegans Excretory Canal Cell as a Model for Tubule Development. Journal of Developmental Biology 8, 17 (2020).

26. Buechner, M. Tubes and the single C. elegans excretory cell. Trends in Cell Biology 12, 479-484 (2002).

27. Kinnunen, T. et al. Heparan 2-O-sulfotransferase, hst-2, is essential for normal cell migration in Caenorhabditis elegans. PNAS 102, 1507-1512 (2005).

28. Cizeron, M., Granger, L., Bülow, H. E. \& Bessereau, J.-L. Specific heparan sulfate modifications stabilize the synaptic organizer MADD-4/Punctin at Caenorhabditis elegans neuromuscular junctions. Genetics 218, (2021).

29. Bülow, H. E. \& Hobert, O. Differential Sulfations and Epimerization Define Heparan Sulfate Specificity in Nervous System Development. Neuron 41, 723-736 (2004).

30. Altun, Z. F. \& Hall, D. H. WormAtlas Hermaphrodite Handbook - Excretory System. WormAtlas (2002) doi:10.3908/wormatlas.1.17.

31. Durbin, R. M. \& University of Cambridge. Studies on the development and organisation of the nervous system of Caenorhabditis elegans. (University of Cambridge, 1987).

32. WormImage Image Details. https://www.wormimage.org/image. $p h p$ ?id=106926\&page=1.

33. Hedgecock, E. M., Culotti, J. G., Hall, D. H. \& Stern, B. D. Genetics of cell and axon migrations in Caenorhabditis elegans. Development 100, 365-382 (1987).

34. Yanowitz, J. L. et al. UNC-39, the C. elegans homolog of the human myotonic dystrophy-associated homeodomain protein Six5, regulates cell motility and differentiation. Developmental Biology 272, 389402 (2004).

35. Packer, J. S. et al. A lineage-resolved molecular atlas of C. elegans embryogenesis at single-cell resolution. Science 365, (2019).

36. Brooks, R., Williamson, R. \& Bass, M. Syndecan-4 independently regulates multiple small GTPases to promote fibroblast migration during wound healing. Small GTPases 3, 73-79 (2012).

37. Bass, M. D. et al. Syndecan-4-dependent Rac1 regulation determines directional migration in response to the extracellular matrix. J Cell Biol 177, 527-538 (2007).

38. Keller-Pinter, A. et al. The phosphomimetic mutation of syndecan-4 binds and inhibits Tiam1 modulating Rac1 activity in PDZ interaction-dependent manner. PLoS One 12, e0187094 (2017).

39. Bülow, H. E. \& Hobert, O. The Molecular Diversity of Glycosaminoglycans Shapes Animal Development. Annual Review of Cell and Developmental Biology 22, 375-407 (2006).

40. Johnson, R. P., Kang, S. H. \& Kramer, J. M. C. elegans dystroglycan DGN-1 functions in epithelia and neurons, but not muscle, and independently of dystrophin. Development 133, 1911-1921 (2006).

41. Mohamed, A. M. \& Chin-Sang, I. D. The C. elegans nck-1 gene encodes two isoforms and is required for neuronal guidance. Developmental Biology 354, 55-66 (2011).

42. Pan, C.-L., Peng, C.-Y., Chen, C.-H. \& Mclntire, S. Genetic analysis of age-dependent defects of the Caenorhabditis elegans touch receptor neurons. Proc. Natl. Acad. Sci. U.S.A. 108, 9274-9279 (2011).

43. Toth, M. L. et al. Neurite Sprouting and Synapse Deterioration in the Aging Caenorhabditis elegans Nervous System. J Neurosci 32, 8778-8790 (2012).

44. Tank, E. M. H., Rodgers, K. E. \& Kenyon, C. Spontaneous Age-Related Neurite Branching in Caenorhabditis elegans. J Neurosci 31, 9279-9288 (2011).

45. Jia, R. et al. Spectrin-based membrane skeleton is asymmetric and remodels during neural development. J Cell Sci (2020) doi:10.1242/jcs.248583.

46. Kirszenblat, L., Neumann, B., Coakley, S. \& Hilliard, M. A. A dominant mutation in mec-7/ß-tubulin affects axon development and regeneration in Caenorhabditis elegans neurons. Mol Biol Cell 24, 285296 (2013).

47. Manon-Jensen, T., Itoh, Y. \& Couchman, J. R. Proteoglycans in health and disease: the multiple roles of syndecan shedding. FEBS J 277, 3876-3889 (2010). 
48. Minniti, A. N., Labarca, M., Hurtado, C. \& Brandan, E. Caenorhabditis elegans syndecan (SDN-1) is required for normal egg laying and associates with the nervous system and the vulva. Journal of Cell Science 117, 5179-5190 (2004).

49. Matsumoto, Y., Irie, F., Inatani, M., Tessier-Lavigne, M. \& Yamaguchi, Y. Netrin-1/DCC Signaling in Commissural Axon Guidance Requires Cell-Autonomous Expression of Heparan Sulfate. J Neurosci 27, 4342-4350 (2007).

50. Inatani, M., Irie, F., Plump, A. S., Tessier-Lavigne, M. \& Yamaguchi, Y. Mammalian Brain Morphogenesis and Midline Axon Guidance Require Heparan Sulfate. Science 302, 1044-1046 (2003).

51. Schwabiuk, M., Coudiere, L. \& Merz, D. C. SDN-1/syndecan regulates growth factor signaling in distal tip cell migrations in C. elegans. Developmental Biology 334, 235-242 (2009).

52. Zhou, X. et al. The HSPG syndecan is a core organizer of cholinergic synapses. J Cell Biol 220, e202011144 (2021).

53. Demarco, R. S., Struckhoff, E. C. \& Lundquist, E. A. The Rac GTP Exchange Factor TIAM-1 Acts with CDC-42 and the Guidance Receptor UNC-40/DCC in Neuronal Protrusion and Axon Guidance. PLoS Genet 8, e1002665 (2012).

54. Levy-Strumpf, N. \& Culotti, J. G. VAB-8, UNC-73 and MIG-2 regulate axon polarity and cell migration functions of UNC-40 in C. elegans. Nature Neuroscience 10, 161-168 (2007). 
Figure 1. Perturbation of HSPGs results in additional cellular projections. (A) Ventral views of fourth larval stage animals by fluorescence microscopy. The excretory canal cell's soma is located ventrally in the head. In the wild type, the excretory cell possesses 4 canals along the animal's body sides, 2 anteriorly and 2 posteriorly. In mutants for the genes rib-1 and rib-2, which encode the HS co-polymerase, the excretory cell displays supernumerary canals (i.e. more than 4 canals). Yellow arrowheads point supernumerary canals. Scale bar, $20 \mu \mathrm{m}$. (B) Schematics of HS chains biosynthesis on HSPG core proteins. Following the addition of a tetrasaccharide linker on specific Ser residues on the core proteins, HS polysaccharide chains are extended by the action of the HS copolymerase, which is composed of RIB-1 and RIB-2 in C. elegans. HS chains are subsequently modified by several modifying enzymes. (C) Quantification of the supernumerary canals of the excretory canal cell defects in hypomorphic mutants for the genes rib-1 and rib-2. (D) Quantification of supernumerary excretory canals defects shown by mutants for genes encoding enzymes responsible of HS chain modifications. (E) Quantification of defective canal number in mutants for genes encoding HSPG core proteins. Animals mutant for sdn-1/syndecan, but not for other HSPGs, have significantly more than four canals. Error bars are standard error of the proportion. Asterisks denote significant difference: ${ }^{*} p \leq 0.05,{ }^{* * *} p \leq 0.001$ (z-tests, $p$-values were corrected by multiplying by the number of comparisons). ns, not significant.

Figure 2. Supernumerary canals of the excretory canal cell displayed by sdn1/Syndecan mutants develop embryonically. (A) Timeline of the excretory canal cell development. During embryonic development, the excretory cell precursor is born at 270 min, followed by tube formation starting at $\sim 400 \mathrm{~min}$, and the formation of two lateral arms at the 1.5-fold stage, which will bifurcate to form four canals at the 2-fold stage. These excretory canals keep extending along the animal's body side until the $4^{\text {th }}$ larval stage. (B) Fluorescence microscopy images of wild-type and sdn-1(zh20) mutant embryos. The excretory cell is visualized with marker Pglt-3::venus. Supernumerary canals are already visible when the normal set of canals develops during embryogenesis. Panels to the right schematize the excretory canal cell with a presumptive supernumerary canals in red as we cannot differentiate it from the normal canals. (C) The supernumerary canal phenotype of $s d n-1$ (zh20) null mutant animals is similar in penetrance at the $1^{\text {st }}(\mathrm{L} 1)$ and $4^{\text {th }}$ (L4) larval stages. (D) Confocal microscopy images of $1^{\text {st }}$ larval stage wild-type and $s d n-1$ (zh20) animals. The cytoplasm of the excretory cell is visualized by the marker Pglt-3::cfp (cyan) and actin by the marker LifeAct::TagRFP (magenta). All the excretory canals, including the supernumerary ones in sdn-1 mutants, display actin apically in a similar manner. Arrowheads point to the tip of each supernumerary canals. Scale bar, 10 $\mu \mathrm{m}$. Error bars are standard error of the proportion. ns, not significant (z-test).

Figure 3. sdn-1/Syndecan acts in a cell-autonomous manner to control canal number during development. (A) Left panel is a transverse view of an approximately 500 min embryo, by transmission electron microscopy (TEM ${ }^{1}$ ). Cells surrounding the excretory cell (red) have been artificially coloured (epidermis in brown, seam cells in beige, body wall muscles in blue, neurons in purple, and excretory duct cell in cyan). Right panel is a schematic representation of the TEM view. (B) The supernumerary canal 
defects displayed by $s d n-1(z h 20)$ null mutant animals are fully rescued by expressing wild-type copies of $s d n-1 /$ syndecan under a heterologous promoter that drives expression specifically in the excretory cell [Pglt-3::sdn-1(+)] indicating that sdn-1 functions cellautonomously to control the number of canals. Expression of wild-type copies of sdn$1 /$ syndecan under other heterologous promoters driving expression in neighbouring tissues did not rescue the defects of sdn-1(zh20) mutants. Error bars are standard error of the proportion. Asterisks denote significant difference compared to sdn-1(zh20) group: *** $p \leq 0.001$ (z-tests, p-values were corrected by multiplying by the number of comparisons). ns, not significant.

Figure 4. Disruption of the UNC-6/Netrin guidance pathway affects the excretory cell canal number. (A) The UNC-6/Netrin signal acts through its main receptors UNC40/DCC and UNC-5/UNC5. (B) Ventral views of $4^{\text {th }}$ larval stage wild-type and mutant animals, where the excretory cell is visualized by expressing Pglt-3::venus. The region observed in these images is indicated by the rectangle on the diagram. Arrowheads point supernumerary canals. Scale bar, $10 \mu \mathrm{m}$. (C) Quantification of supernumerary canals defects in mutants for genes encoding guidance cue molecules or their receptors. Null mutant animals unc-6(ev400), unc-40(e1430) and unc-5(e53) show supernumerary canals. (D) Quantification of supernumerary canals defects in animals doubly mutants for syndecan/sdn-1(zh20) and genes encoding guidance receptors, unc-40(e1430) or unc5(e53). The fact that these double mutants are not more severe than the single mutant sdn-1(zh20) suggests that the genes function in the same pathway. Error bars are standard error of the proportion. Asterisks denote significant difference: ${ }^{* * *} p \leq 0.001,{ }^{* *}$ $p \leq 0.01$ and * $p \leq 0.05$ (z-tests, $p$-values were corrected by multiplying by the number of comparisons). ns, not significant.

Figure 5. The control of the number of canals requires Rho GTPases. (A) Quantification of supernumerary canal defects in mutants of ced-10. Alone, the ced10(n1993) loss-of-function mutants do not induce extra canals, but suppresses the canal number defects sdn-1(zh20) null mutants. (B) Quantification of supernumerary canal defects in mutants of mig-2. Single null mutants mig-2(mu28) alone does not display supernumerary canals, but suppresses the canal number defects sdn-1(zh20) null mutants. (C) Schematic summary of molecules implicated in controlling cellular projection number and possible mechanisms. SDN-1/Syndecan is central HSPG to control the number of canals in the excretory cell and could coordinate signals from the UNC-6/Netrin guidance pathway to drive proper cell development. The mechanism under the scope may converge onto RhoGTPases to regulate canal number. Error bars are standard error of the proportion. Asterisks denote significant difference: ${ }^{* *} p \leq 0.001$ (z-tests, p-values were corrected by multiplying by the number of comparisons). ns, not significant. 
A
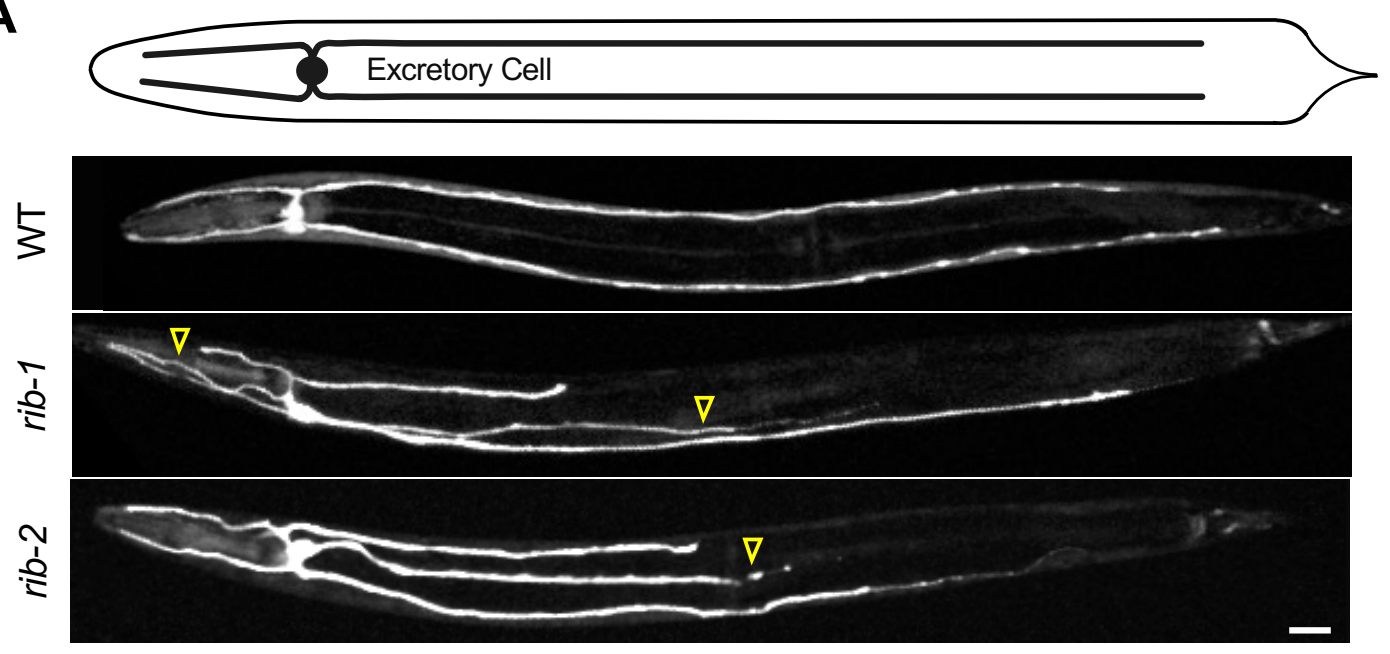

B

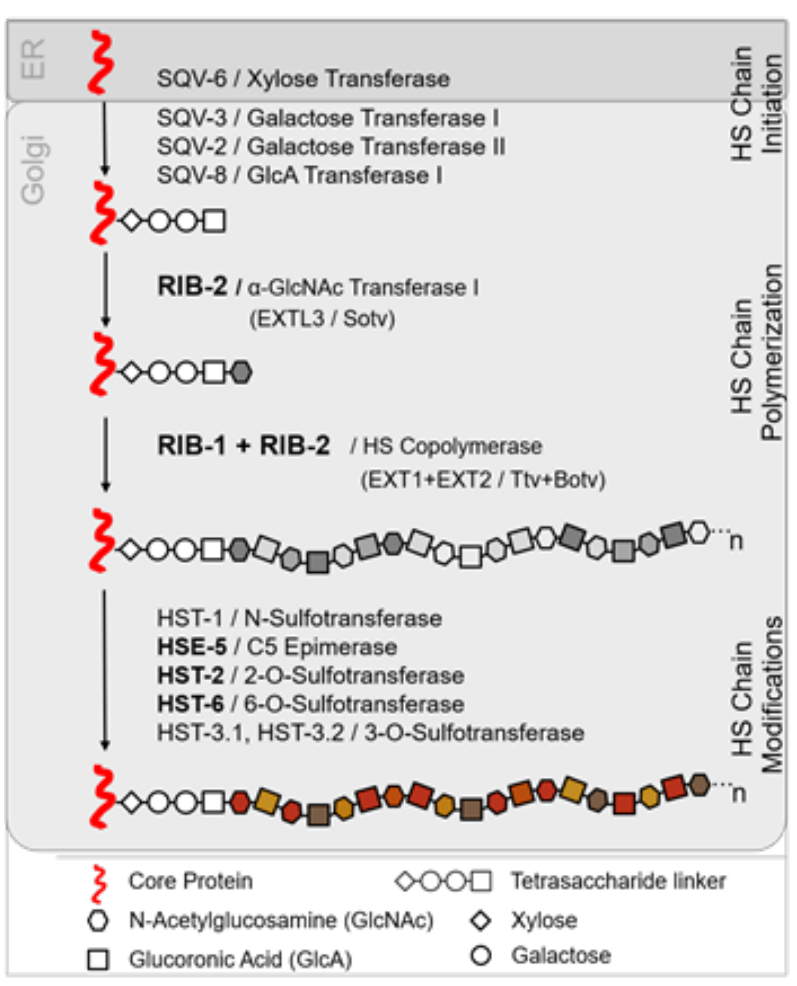

E

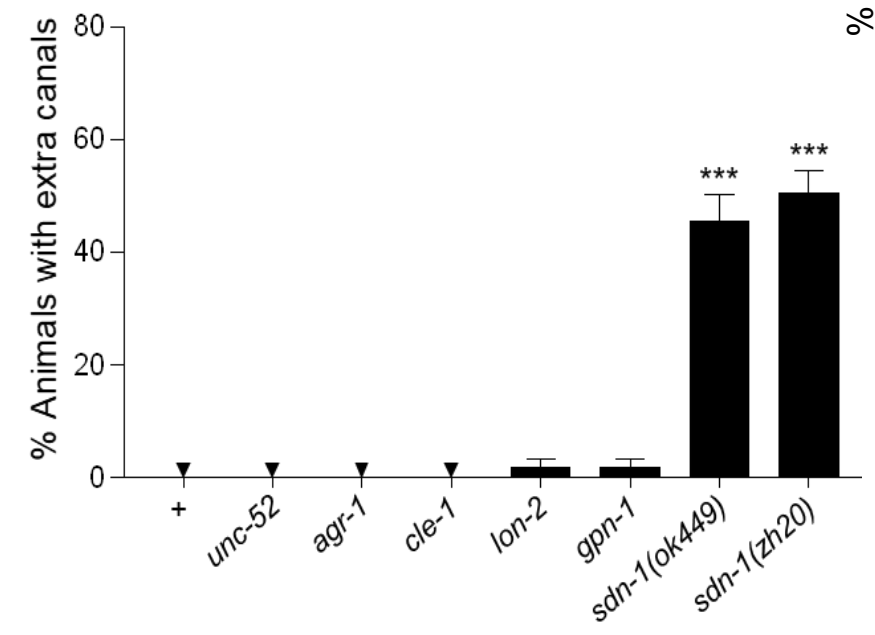

C

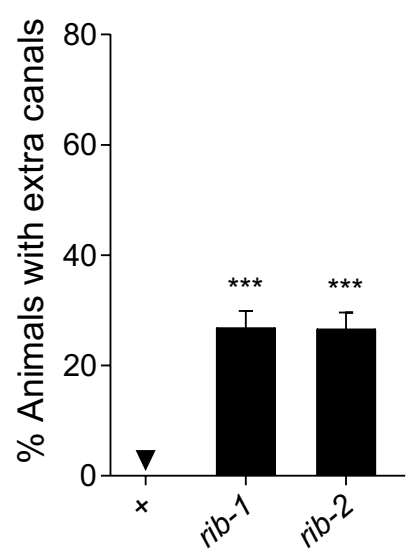

D

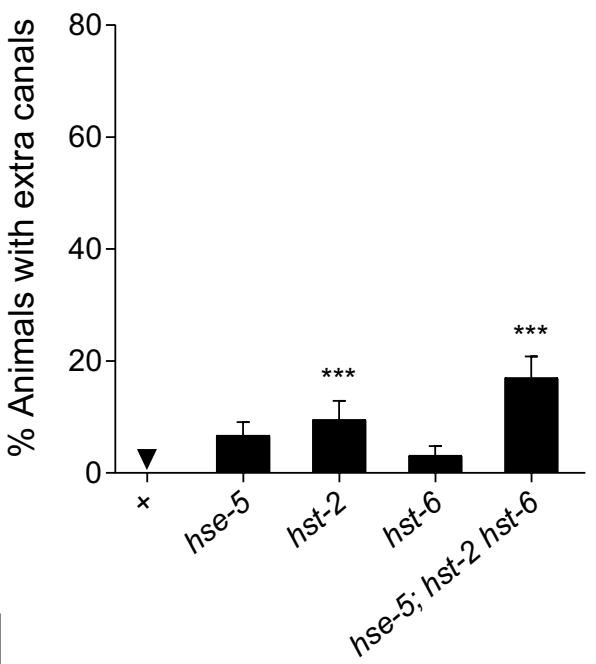


A

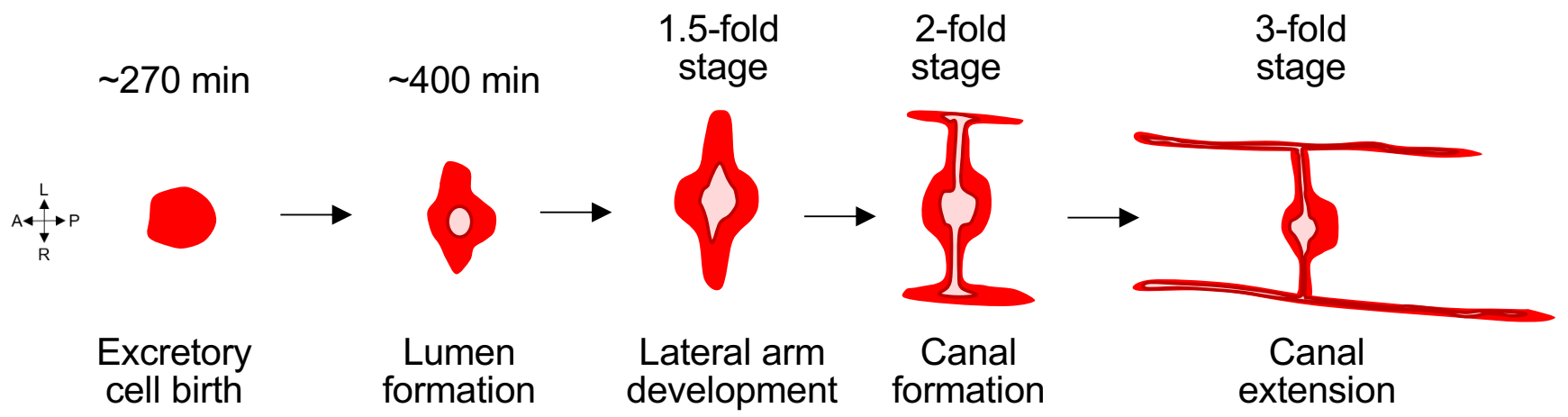

B

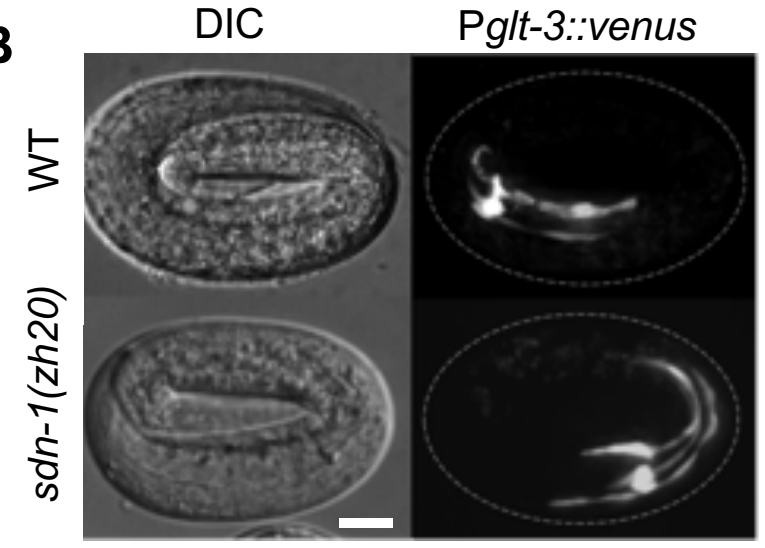

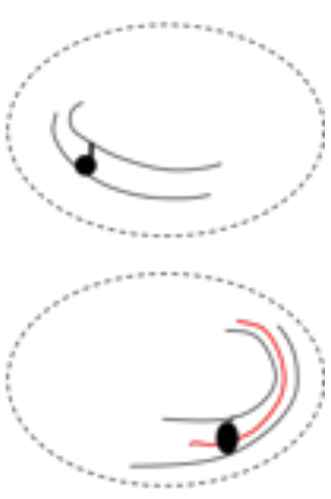

C

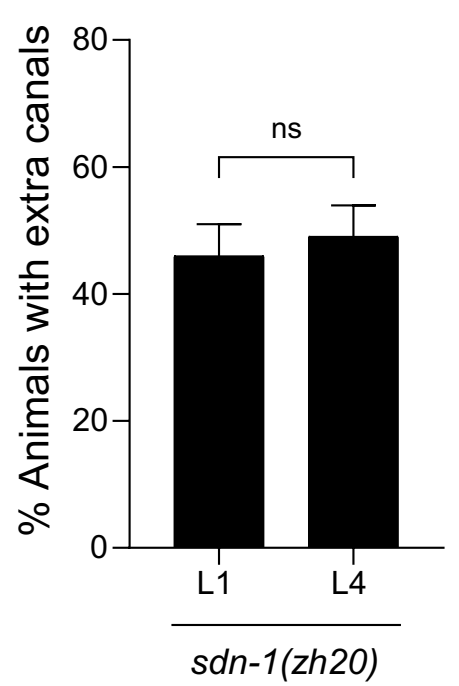

D

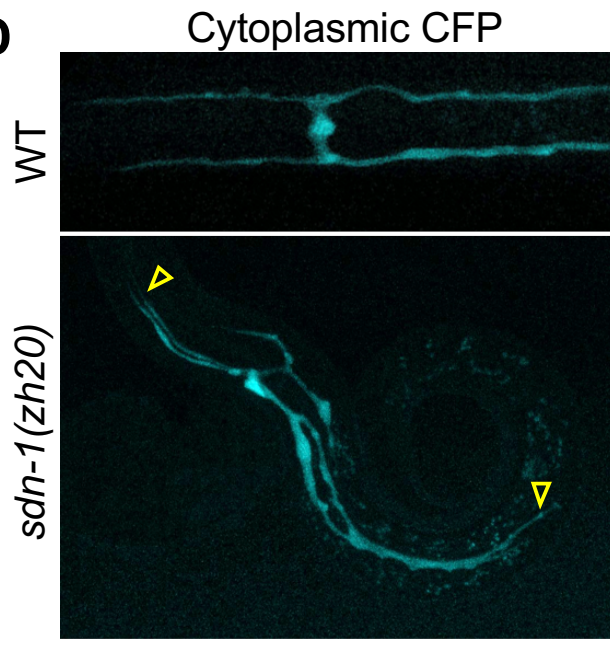

LifeAct::TagRFP

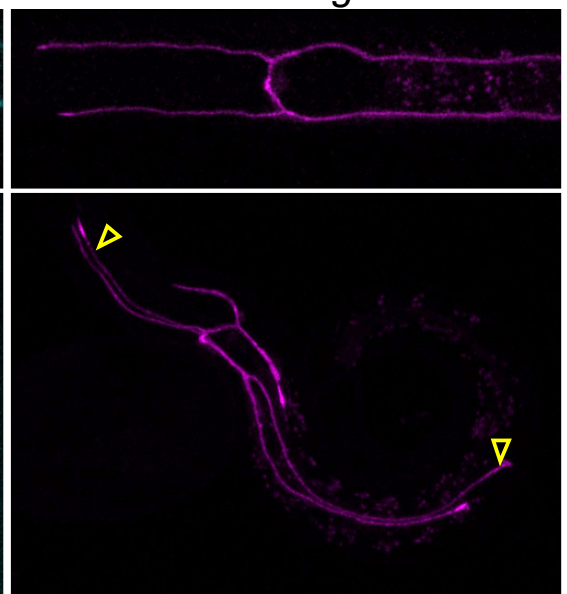

Merge

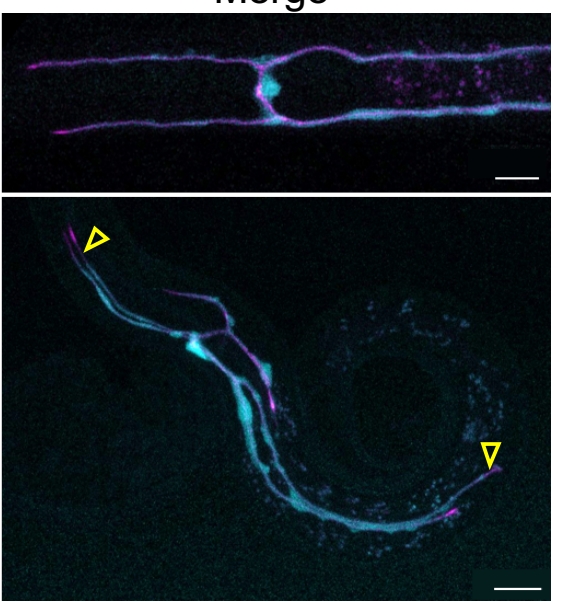


A
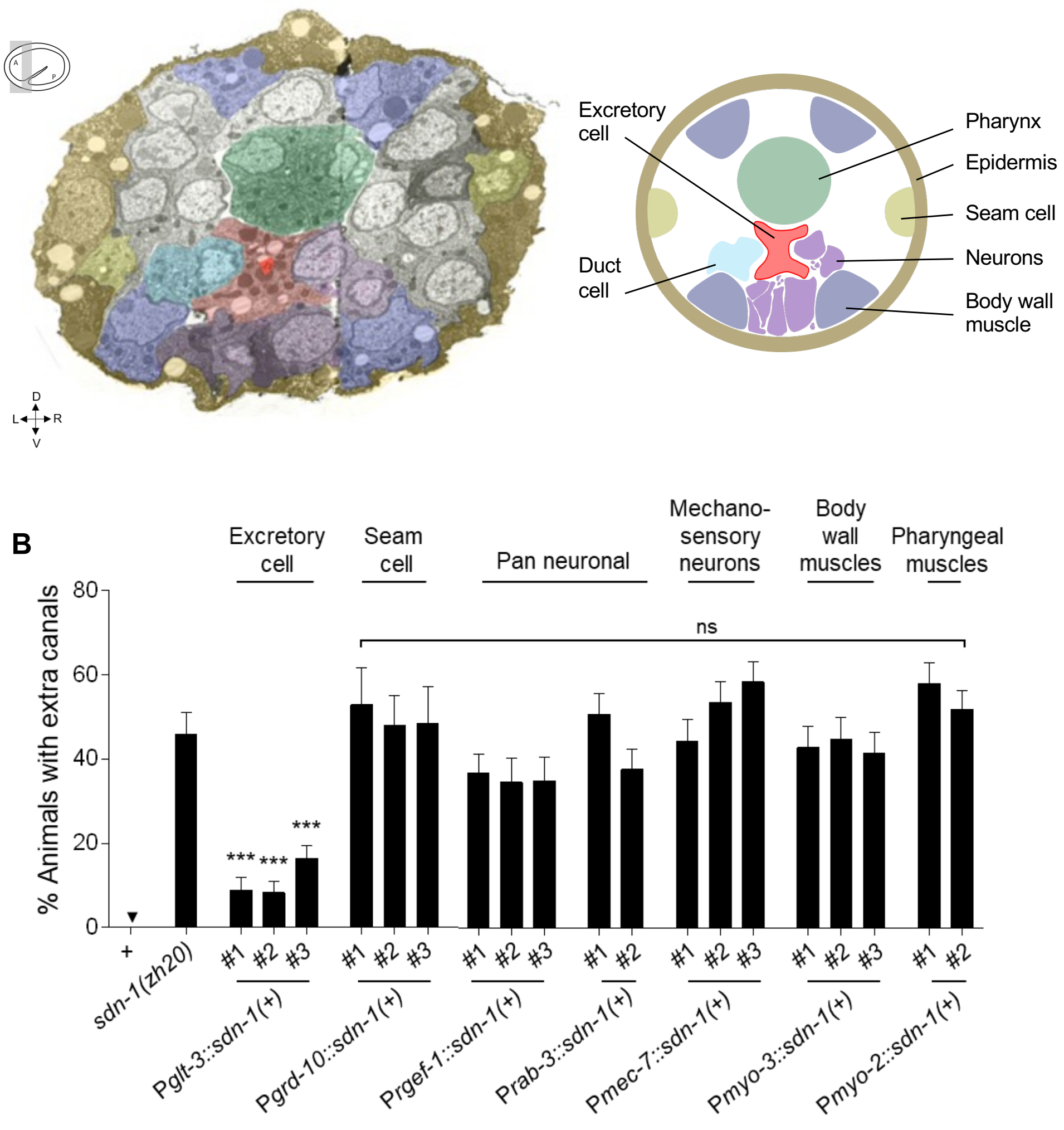

sdn-1(zh20) 
Figure 4
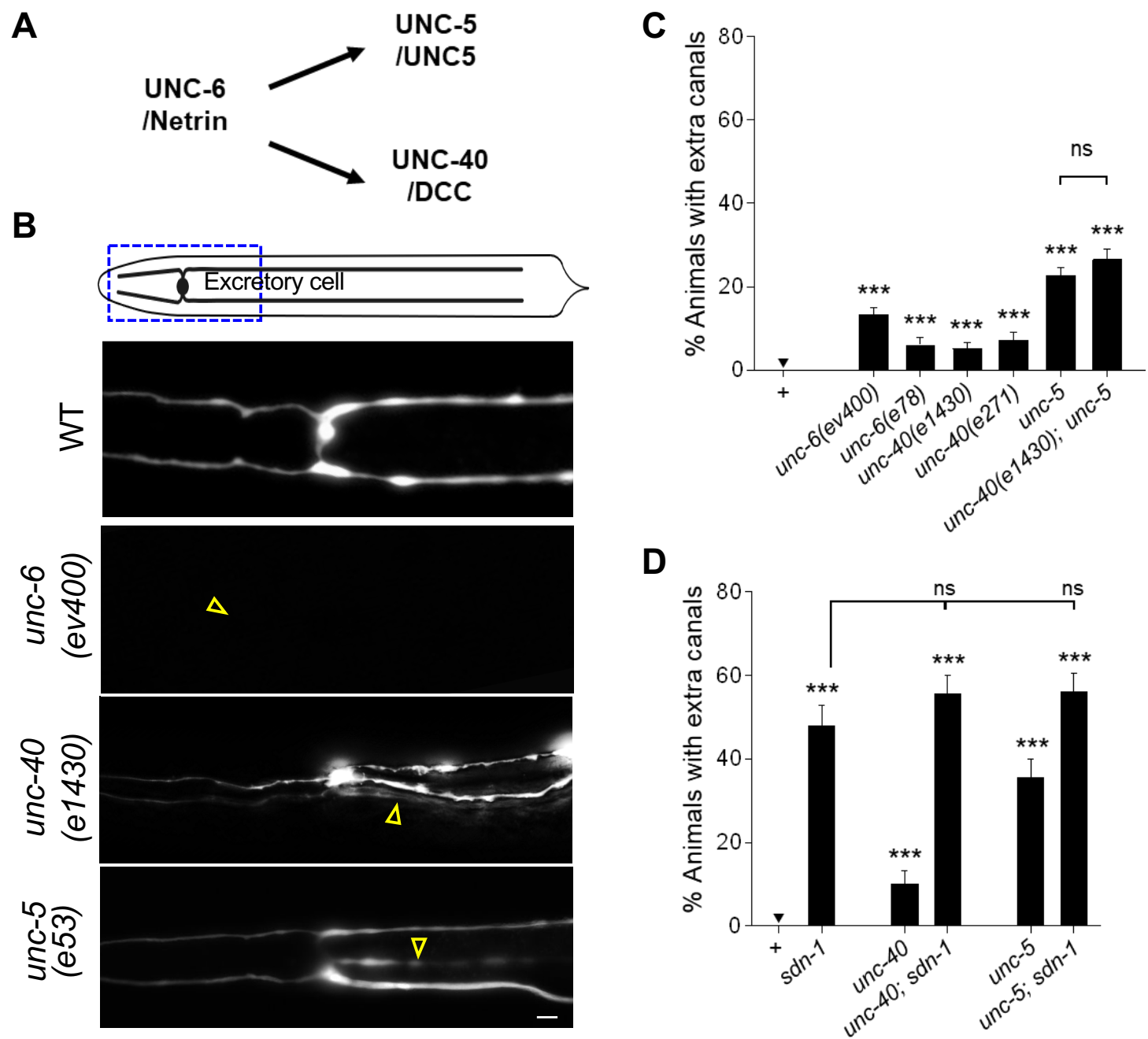

D

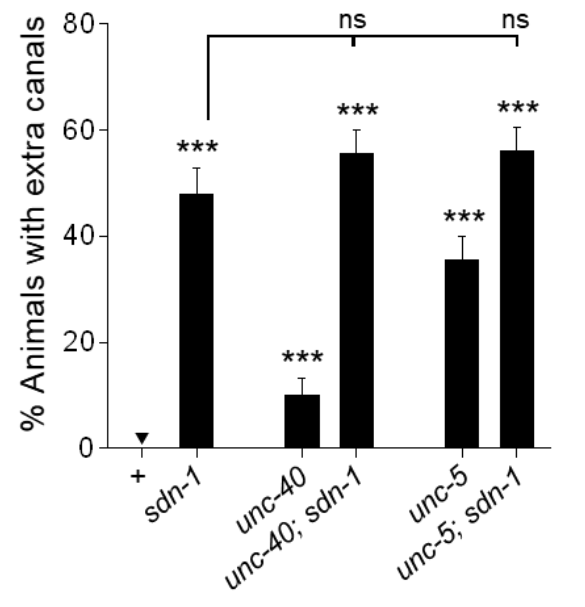


Figure 5

A

C

(1)
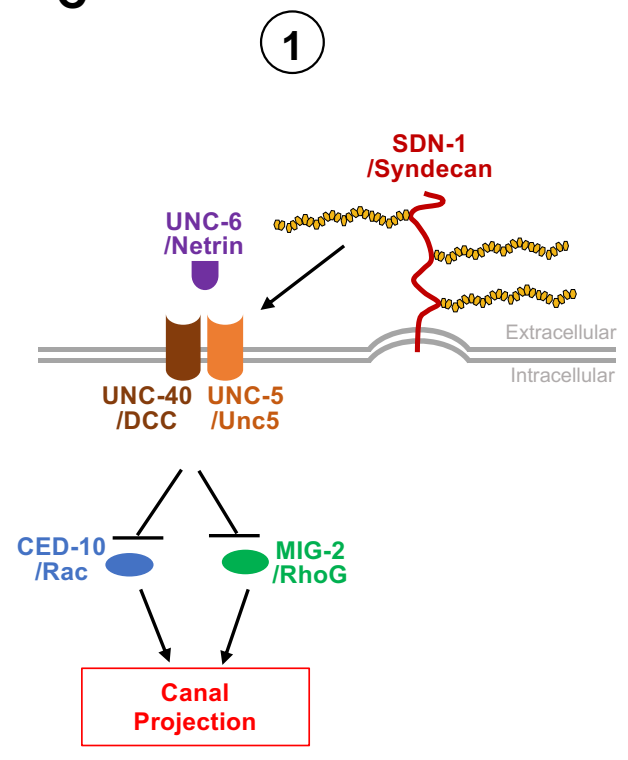

B

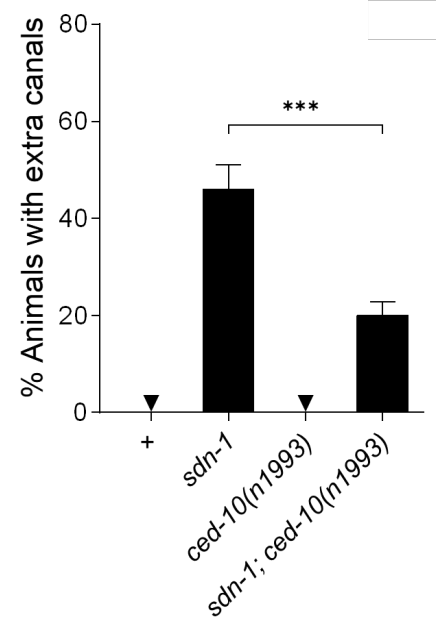

B

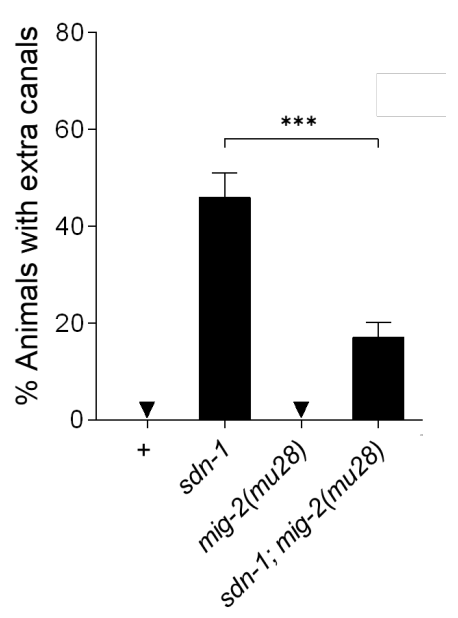

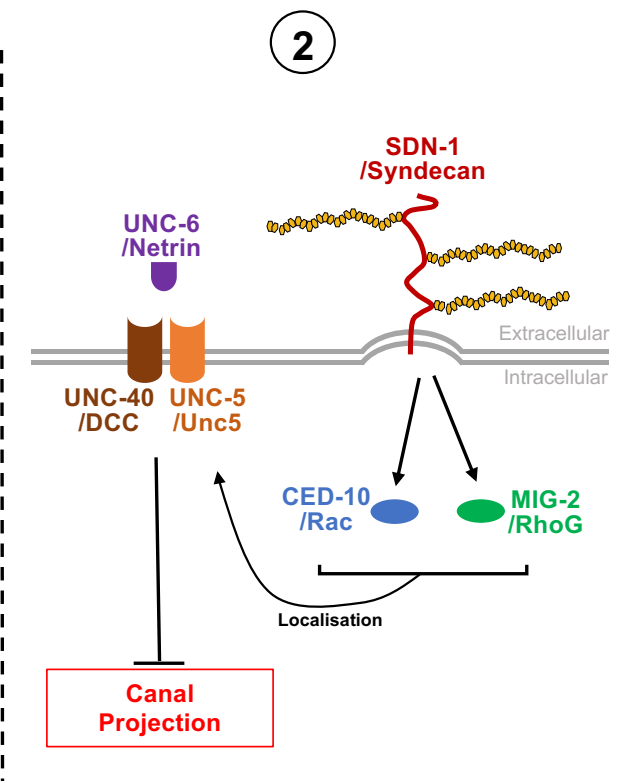

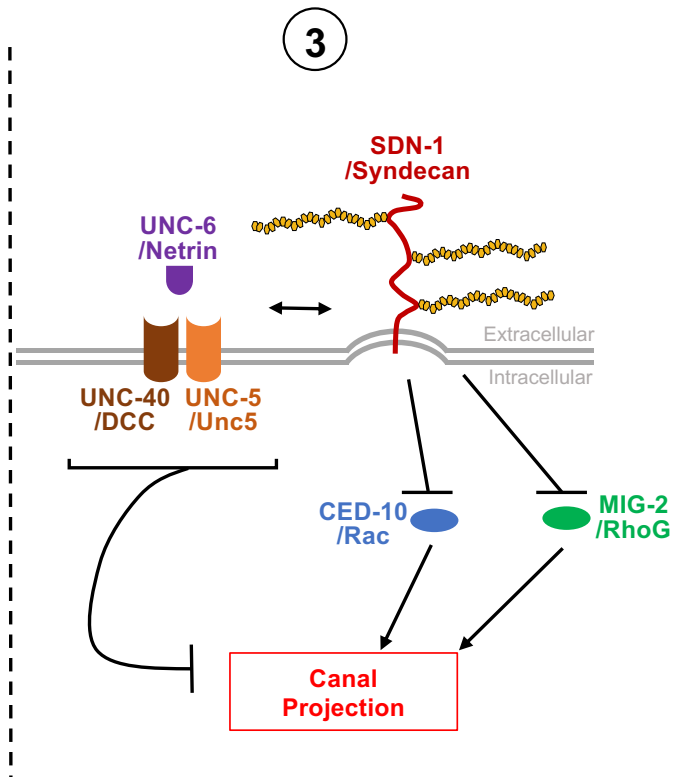

